\title{
SEXUAL HEALTH, AIDS-HIV, SEXUALLY TRANSMISSIBLE INFECTION COORDINATORS CONTACT LIST
}

\begin{tabular}{|c|c|c|c|c|c|}
\hline Area Health Service & Contact & Mailing Address & Email & Phone & Fax \\
\hline Central Coast & Ken Wong & $\begin{array}{l}\text { PO Box } 361 \\
\text { Gosford NSW } 2250\end{array}$ & kwong@doh.health.nsw.gov.au & $\begin{array}{l}\text { (02) } 43202114 \text { or } \\
\text { (02) } 43203399\end{array}$ & (02) 43203163 \\
\hline \multirow[t]{2}{*}{ Central Sydney } & Peter Todaro & $\begin{array}{l}\text { Level 5, Queen Mary Bldg } \\
\text { Grose Street, Camperdown NSW } 2050\end{array}$ & ptoda@hiv.rpa.cs.nsw.gov.au & $\begin{array}{l}\text { (02) } 95153202 \text { or } \\
\text { (0413) } 017613\end{array}$ & (02) 95153379 \\
\hline & $\begin{array}{l}\text { Janice Pritchard-Jones } \\
\text { Hepatitis C Nurse } \\
\text { Consultant }\end{array}$ & $\begin{array}{l}\text { Gastroenterlogy Unit } \\
\text { Royal Prince Alfred } \\
\text { Hospital, Missenden Road } \\
\text { Camperdown NSW } 2050\end{array}$ & jonesj@rpamail.cs.nsw.gov.au & (02) 95158643 & (02) 95158242 \\
\hline Corrections Health & Debbie Pittam & Private Bag 155, Silverwater NSW 1811 & $\mathrm{n} / \mathrm{a}$ & $(0419) 429300$ & (02) 92895981 \\
\hline Far West & Darriea Turley & $\begin{array}{l}\text { PO Box } 457 \\
\text { Broken Hill NSW } 2880\end{array}$ & $\mathrm{n} / \mathrm{a}$ & $\begin{array}{l}(08) 80801511 \text { or } \\
(0419) 975976\end{array}$ & (08) 80878697 \\
\hline Greater Murray & Dalton Dupuy & $\begin{array}{l}\text { PO Box } 503 \\
\text { Albury NSW } 2640\end{array}$ & dalton.dupuy@swsahs.nsw.gov.au & $\begin{array}{l}(02) 60581731 \text { or } \\
(0427) 480271\end{array}$ & (02) 60581737 \\
\hline Hunter & Marilyn Bliss & PO Box 466, Wallsend NSW 2287 & mblis@doh.health.nsw.gov.au & (02) 49246477 & (02) 49246490 \\
\hline Illawarra & Brian O’Neill & $\begin{array}{l}\text { Suite 5, } 5 \text { Rawson Street } \\
\text { Wollongong NSW } 2500\end{array}$ & oneillb@iahs.nsw.gov.au & (02) 42288033 & (02) 42252177 \\
\hline Macquarie & Scott Davis & $\begin{array}{l}194 \text { Brisbane Street } \\
\text { Dubbo NSW } 2830\end{array}$ & scadvis@doh.health.nsw.gov.au & (02) 68851700 & (02) 68844315 \\
\hline Mid North Coast & Robert Baldwin & $\begin{array}{l}\text { PO Box } 126 \\
\text { Port Macquarie NSW } 2444\end{array}$ & rbald@doh.health.nsw.gov.au & $\begin{array}{l}(02) 65882750 \text { or } \\
(0417) 268055\end{array}$ & (02) 65882837 \\
\hline Mid Western & David Brackenreg & $\begin{array}{l}\text { PO Box } 143 \\
\text { Bathurst NSW } 2795\end{array}$ & $\mathrm{n} / \mathrm{a}$ & $\begin{array}{l}(02) 63395576 \text { or } \\
(0413) 186978\end{array}$ & (02) 63395555 \\
\hline New England & Karin Fisher & PO Box 597, Tamworth NSW 2340 & kfish@doh.health.nsw.gov.au & $(02) 67662288$ & (02) 67663003 \\
\hline Northern Rivers & Wendi Evans & Locked Bag 11, Lismore NSW 2480 & wevan@nrhs.health.nsw.gov.au & (02) 66207505 & (02) 66217088 \\
\hline Northern Sydney & Graham Stone & $\begin{array}{l}\text { Executive Office, Main Block, } \\
\text { Royal North Shore Hospital, } \\
\text { Pacific Highway, } \\
\text { St Leonards NSW } 2065\end{array}$ & $\mathrm{n} / \mathrm{a}$ & (02) 99266717 & (02) 99266710 \\
\hline South Eastern Sydney & Philip Jones & $\begin{array}{l}\text { Royal South Sydney } \\
\text { Community Health Centre, } \\
\text { Joynton Avenue, Zetland NSW } 2017\end{array}$ & jonesp@sesahs.nsw.gov.au & (02) 93823405 & (02) 93823403 \\
\hline Southern NSW & Geetha Isaac-Toua & $\begin{array}{l}\text { Sexual Health Service } \\
\text { Locked Bag } 11 \\
\text { Goulburn NSW } 2580\end{array}$ & gisaa@doh.health.nsw.gov.au & $\begin{array}{l}(02) 48273428 \text { or } \\
(02) 62958330 \text { or } \\
(0410) 695832\end{array}$ & $\begin{array}{l}\text { (02) } 48273438 \text { or } \\
\text { (02) } 62958330\end{array}$ \\
\hline South Western Sydney & James Mabbut & $\begin{array}{l}\text { Locked Bag } 7017 \\
\text { Liverpool BC NSW } 1871\end{array}$ & James.Mabbut@swsahs.nsw.gov.au & (02) 98285944 & (02) 98285955 \\
\hline Wentworth & Elizabeth O'Neil & $\begin{array}{l}\text { PO Box } 126 \\
\text { Penrith NSW } 2751\end{array}$ & o'neile@wahs.nsw.gov.au & $\begin{array}{l}\text { (02) } 47343855 \text { or } \\
(02) 47343877\end{array}$ & (02) 47343865 \\
\hline Western Sydney & Chris O'Reilly & $\begin{array}{l}\text { Locked Bag 7118, } \\
\text { Parramatta BC NSW } 2150\end{array}$ & chris_oreilly@wsahs.nsw.gov.au & (02) 98404105 & (02) 98404104 \\
\hline
\end{tabular}

